\title{
A 40,000-YEAR VARVE CHRONOLOGY FROM LAKE SUIGETSU, JAPAN: EXTENSION OF THE ${ }^{14} \mathrm{C}$ CALIBRATION CURVE
}

\author{
HIROYUKI KITAGAWA ${ }^{1}$ and JOHANNES VAN DER PLICHT ${ }^{2}$
}

\begin{abstract}
A sequence of annually laminated sediments is a potential tool for calibrating the radiocarbon time scale beyond the range of the absolute tree-ring calibration $\left(11 \mathrm{ka}\right.$ ). We performed accelerator mass spectrometric (AMS) ${ }^{14} \mathrm{C}$ measurements on $>250$ terrestrial macrofossil samples from a 40,000-yr varve sequence from Lake Suigetsu, Japan. The results yield the first calibration curve for the total range of the ${ }^{14} \mathrm{C}$ dating method.
\end{abstract}

\section{INTRODUCTION}

Lake Suigetsu is located near the coast of the Sea of Japan $\left(35^{\circ} 35^{\prime} \mathrm{N}, 135^{\circ} 53^{\prime} \mathrm{E}\right)$. The lake is $10 \mathrm{~km}$ around the perimeter and covers $4.3 \mathrm{~km}^{2}$. It is a typical kettle-type lake, nearly flat at the center, $c a$. $34 \mathrm{~m}$ deep. A 75-m-long continuous core (Lab code = SG) and four short piston cores (Lab codes = SG1, $-2,-3$ and -4) were taken from the center of the lake before 1993 (Kitagawa et al. 1995).

The sediments are characterized by dark-colored clay with white layers due to spring season diatom growth. The seasonal changes in the depositions are preserved in the clay as thin, sub-millimeter scale laminations or "varves". Based on observation of varve thickness change, we expect that the annually laminated sediment records the paleoenvironmental changes during the past $100 \mathrm{ka}$.

This sequence of annually laminated sediments not only forms a unique continuous paleoenvironmental record after the last interglacial but also permits us to reconstruct a complete ${ }^{14} \mathrm{C}$ calibration extending back to at least $40 \mathrm{ka} \mathrm{BP}$, and probably even more by means of combined isotope enrichment and AMS ${ }^{14} \mathrm{C}$ dating (Kitagawa and van der Plicht 1997).

We have performed AMS ${ }^{14} \mathrm{C}$ measurements on $>250$ terrestrial macrofossil samples of the annual laminated sediments from Lake Suigetsu. Here, we report varve and ${ }^{14} \mathrm{C}$ chronologies of these sediments. The combined varve and ${ }^{14} \mathrm{C}$ chronologies back to $40,000 \mathrm{BP}$ are used to reconstruct a ${ }^{14} \mathrm{C}$ calibration curve for the total range of the ${ }^{14} \mathrm{C}$ dating method.

\section{METHODS}

In order to build up a calendar time scale (i.e., varve chronology) for the Suigetsu (SG) core, a total of 85 subsamples were taken in a section of SG extending from 10.42 to $30.45 \mathrm{~m}$ below the top sediment, each $c a .25 \mathrm{~cm}$ in length, including a $1.5 \mathrm{~cm}$ overlap with neighboring subsamples. To allow detailed observation of the sediments, the well-cleaned surfaces of sediments were scanned with a digital camera with a resolution of $c a .1200$ data points per inch. The $c a .1500$ digital images were processed using an image analyzing program.

Based on a more detailed analysis of the varve sediments, the previous chronology obtained mainly from the short piston cores (Kitagawa et al. 1995) is revised for two reasons: 1) a more precise matching of the floating Lake Suigetsu varve chronology to the available dendrochronologies with a high-resolution $\mathrm{AMS}{ }^{14} \mathrm{C}$ data set, and 2) an updated varve chronology due to previous miscounting of varve numbers. We had identified the white and diatom-rich layers under a microscope with a UV light source. The white layers typically observed in the Holocene and at limited time intervals in the Glacial are easily identified by this procedure. However, after reassessment of varve counting

\footnotetext{
${ }^{1}$ International Research Center for Japanese Studies, 3-2 Oeyama Goryo Nishikyo-ku, Kyoto 610-11 Japan

${ }^{2}$ Centre for Isotope Research, University of Groningen, Nijenborgh 4, NL-9747 AG Groningen, The Netherlands
} 
by means of computer image analysis of digital pictures, we found that the much less distinct varves observed in some intervals during the deglaciation and Glacial could be determined only with a relatively large error. In order to reconstruct a more precise and longer varve chronology for the laminated sediments from Lake Suigetsu, we have reassessed the varve chronology in the whole section during the deglaciation as well as the Glacial up to a depth of $30.45 \mathrm{~m}$.

The uncertainty in the varve chronology comes from two sources: core sampling and varve counting. The SG core parts were divided into $90-\mathrm{cm}$-long sections for sampling from one drilling hole; therefore, there is a potential loss of sediment or varves between samplings. Detailed comparison with short piston cores (SG3 and SG4) for the upper $16 \mathrm{~m}$ shows that the sampling does not cause significant loss of varves-typically $0-2 \mathrm{~cm}$ to a maximum of $3 \mathrm{~cm}$, corresponding to ca. 20-30 yr in the Holocene and ca. $50 \mathrm{yr}$ in the Glacial. Since the varve ages from below $18 \mathrm{~m}$ (corresponding to $c a .20,000 \mathrm{cal} \mathrm{BP}$ ) were estimated by varve counting of a single core, the ages quoted in this paper should be considered as minimum ages, the error increasing with depth.

Since the detectability of the varve depends on the quality of the lamination, it is not straightforward to estimate the accuracy of the varve counting process. Based on the results of some duplicated countings of selected subsamples and independent counting of different subsamples collected from the same horizon, we estimate that the counting error is $<1.5 \%$, corresponding to $150 \mathrm{yr}$ for 10,000 varve yr.

The sediment from the core top to $19.3 \mathrm{~m}$ of SG was split $c a$. every $3 \mathrm{~cm}$ (corresponding to 20-50 yr). The macrofossils were washed out from $c a .60 \mathrm{~cm}^{3}$ of sediment. For the deeper part, the relatively large macrofossils were picked up by hand in a dust-free room to reduce contamination from the surroundings. We selected terrestrial-origin macrofossils such as leaves, branches, and insects for AMS ${ }^{14} \mathrm{C}$ measurements.

The ${ }^{14} \mathrm{C} /{ }^{12} \mathrm{C}$ and ${ }^{13} \mathrm{C} /{ }^{12} \mathrm{C}$ ratios of terrestrial macrofossils were measured at the Groningen AMS facility (van der Plicht et al. 1995; Gottdang, Mous and van der Plicht 1995; Wijma and van der Plicht 1997). An essential procedure is a strict elimination of possible contamination during the sample collection and handling (Wohlfarth et al. 1993). The samples are processed using a strong acid-alkaliacid (AAA) treatment (Mook and Streurman 1983) for both samples and reference blank materials. The reference blank consists of $>50{ }^{14} \mathrm{C}$-free plant materials collected from the deep layer of the same core (corresponding to an age of ca. 90-100 ka). The blank correction is $0.28 \pm 0.03(1 \sigma)$ pMC on average for relatively large samples (containing $>0.7 \mathrm{mg}$ carbon), corresponding to $47,000 \mathrm{BP}$. For smaller samples, the blank level and its scatter increase with decreasing sample size. For age calculation, we include this effect as well as the scatter in 5-6 standards per measurement batch, prepared independently from the HOxII international standard, which is typically $0.5 \%$.

\section{RESULTS}

Figure 1 shows the varve and ${ }^{14} \mathrm{C}$ chronologies as a function of depth of the SG core. Until now, the varve numbers have been counted in the 10.42-30.45 m deep section. The Lake Suigetsu floating varve chronology consists of 29,100 varves. As shown in Figure 1, the sedimentation or annual varve thickness is relatively uniform (typically $1.2 \mathrm{~mm} \mathrm{yr}^{-1}$ during the Holocene and $0.62 \mathrm{~mm} \mathrm{yr}^{-1}$ during the Glacial). The age below $30.45 \mathrm{~m}$ depth is obtained by assuming a constant sedimentation

* in the Glacial $\left(0.62 \mathrm{~mm} \mathrm{yr}^{-1}\right)$. The ${ }^{14} \mathrm{C}$ ages at $10.42,30.45$ and $35 \mathrm{~m}$ depth are $c a .7800,35,000$ and $42,000 \mathrm{BP}$, respectively. 


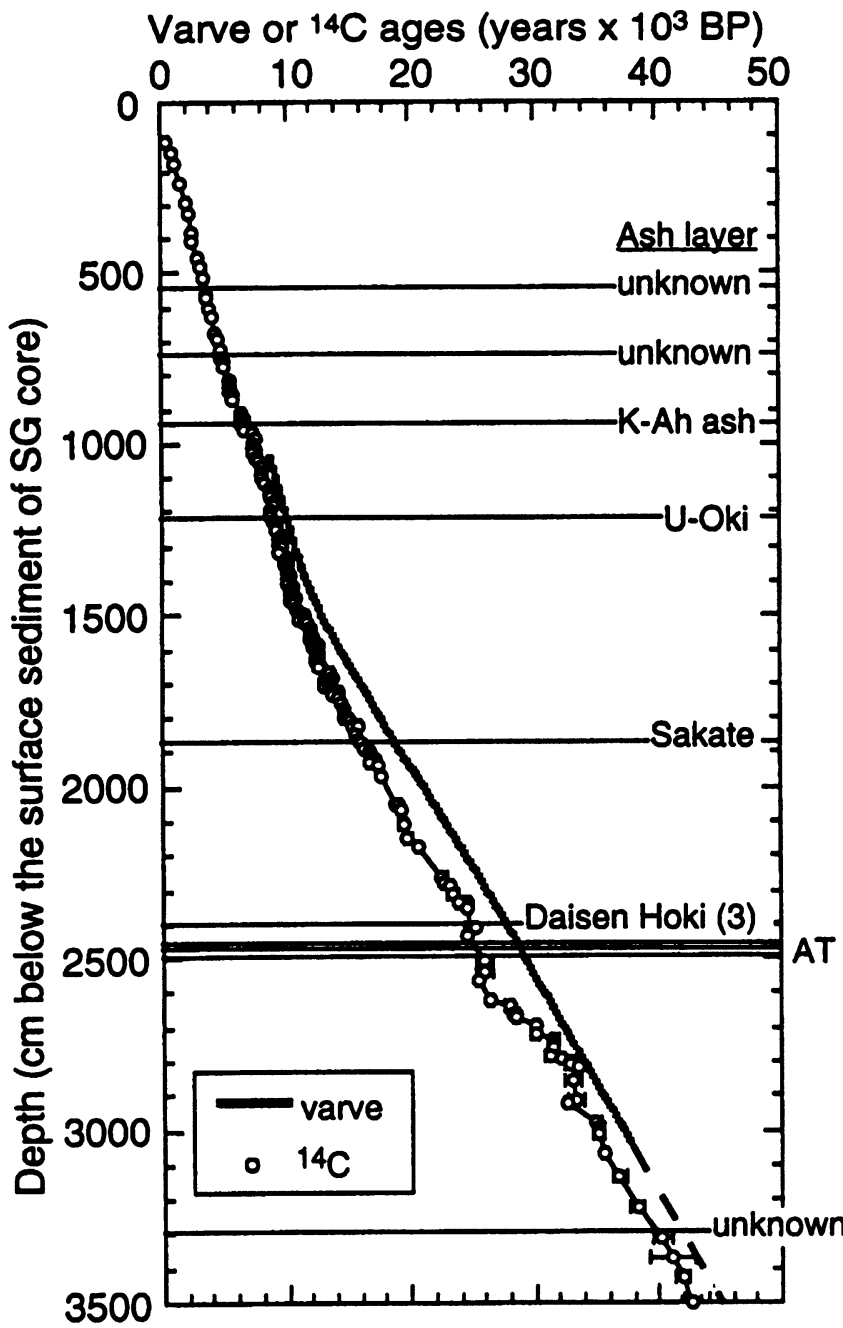

Fig. 1. Varve and radiocarbon chronologies of Lake Suigetsu (SG) core. The ash layers are found in this core section up to $35 \mathrm{~m}$ (Takemura et al. 1994).

In order to reconstruct the calendar time scale, we compared the Lake Suigetsu chronology with calibration curves obtained from the absolute German oak (shifted by $41 \mathrm{yr}$ at $5241 \mathrm{BC}$ to the older direction, Kromer et al. 1996) and the floating German pine (Kromer and Becker 1993) using the least squares minimization. The revised German oak and the floating German pine calibration curves were combined into one calibration curve by moving the age of German pine chronology.

Figure 2 shows the best match between the tree-ring and the Lake Suigetsu chronologies, estimated by minimizing the weighted sum of squared differences between the ${ }^{14} \mathrm{C}$ ages of macrofossils and the tree-ring calibration curve. We found the best match when the German pine chronology is shifted by $160 \mathrm{yr}$ with respect to the pine chronology reported by Kromer and Becker (1993). The features in our data overlapping the tree ring calibration agree very well, even for "wiggles" in the ${ }^{14} \mathrm{C}$ calibration curves. Using this match, we defined the absolute time scale for the Lake Suigetsu varves chronology. The 29,100-yr Lake Suigetsu chronology then covers the absolute age range from 8830 


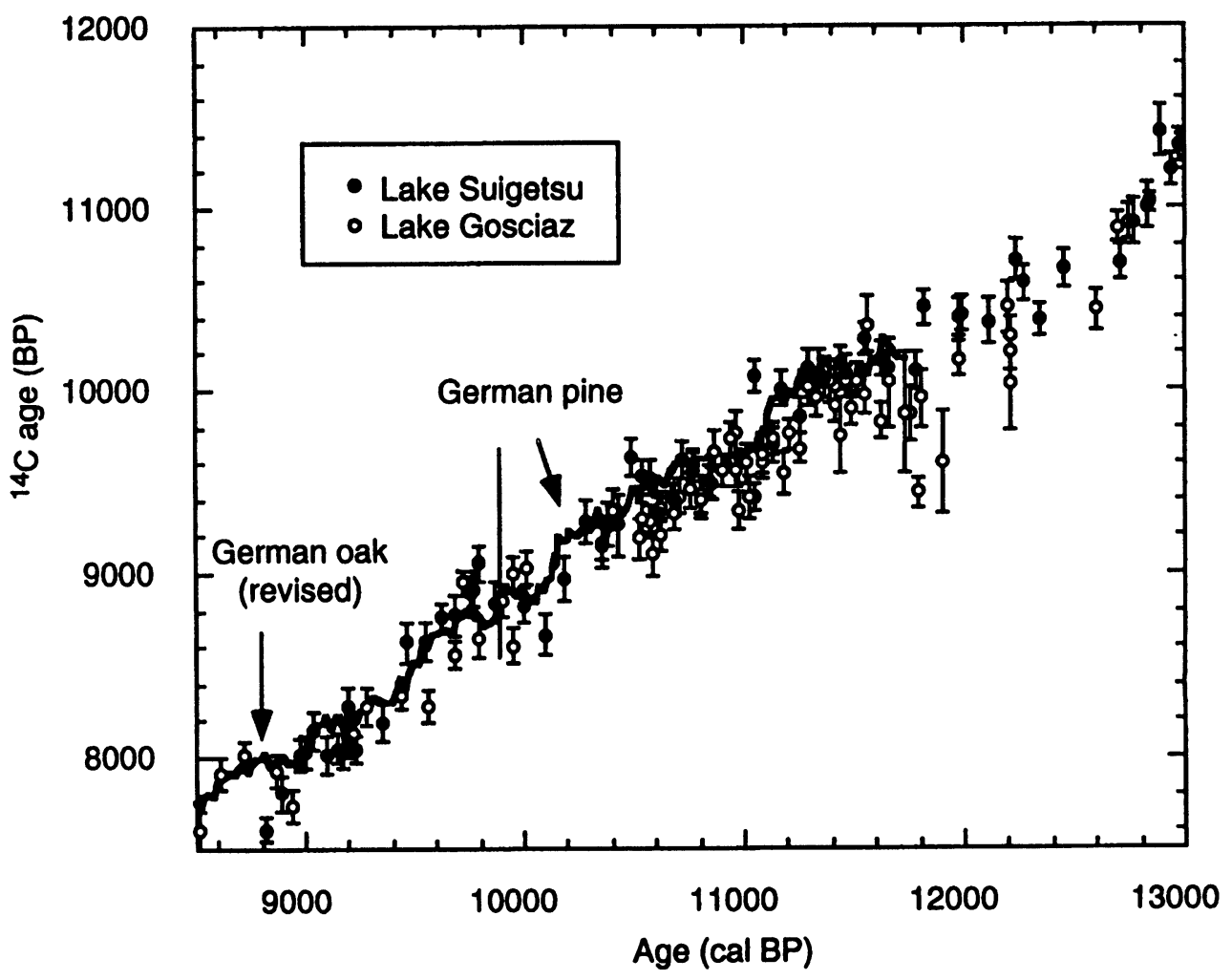

Fig. 2. Matching of the 29,100-yr-long floating varve chronology from Lake Suigetsu to the absolute chronology. = Lake Suigetsu (Japan); 0 = Lake Gosciaz (Poland) (Goslar et al. 1995, ms.). Continuous lines show the German oak and pine chronologies fixed by comparison with the varve chronology of Lake Suigetsu.

to 37,930 cal BP. Our varve chronology also confirms the revised floating German pine chronology, which was recently shifted by $160 \mathrm{yr}$ to the older direction (Björck et al. 1996; Kromer et al. 1996).

The combined ${ }^{14} \mathrm{C}$ and varve chronologies from Lake Suigetsu are used to calibrate the ${ }^{14} \mathrm{C}$ time scale beyond the range of the absolute tree-ring calibration. Figure 3 shows an atmospheric ${ }^{14} \mathrm{C} \mathrm{cal}-$ ibration for the complete ${ }^{14} \mathrm{C}$ dating range $(<45 \mathrm{ka}$ ) reconstructed from annually laminated sediments from Lake Suigetsu. The numbers are given in the Appendix. Beyond the-tree ring calibration range, our calibration agrees well with the European sediments (Goslar et al. 1995, Goslar, Arnold and Tisnerat-Laborde ms.) and generally with marine calibrations obtained by combined U/Th and ${ }^{14} \mathrm{C}$ dating of corals (Bard et al. 1990, 1993; Edwards et al. 1993). Our data confirms the higher ${ }^{14} \mathrm{C}$ levels for the Glacial period likely induced by a low geomagnetic field intensity (e.g., Laj, Mazaud and Duplessy 1996). The maximum difference between ${ }^{14} \mathrm{C}$ and calendar ages during the past $45 \mathrm{ka}$ is ca. $5000 \mathrm{yr}$ around $30,000 \mathrm{cal} \mathrm{BP}$. Similar data are obtained by Voelker et al. (1998).

\section{CONCLUSION}

The atmospheric ${ }^{14} \mathrm{C}$ concentration is sensitive to parameters such as geomagnetic field strength and solar fluctuations (also through magnetic effects) as well as rearrangements in equilibrium between the major carbon reservoirs (atmosphere, ocean and biosphere). High-resolution ${ }^{14} \mathrm{C}$ calibration extending into the Glacial is therefore critical for establishing the exact timing of drastic cli- 


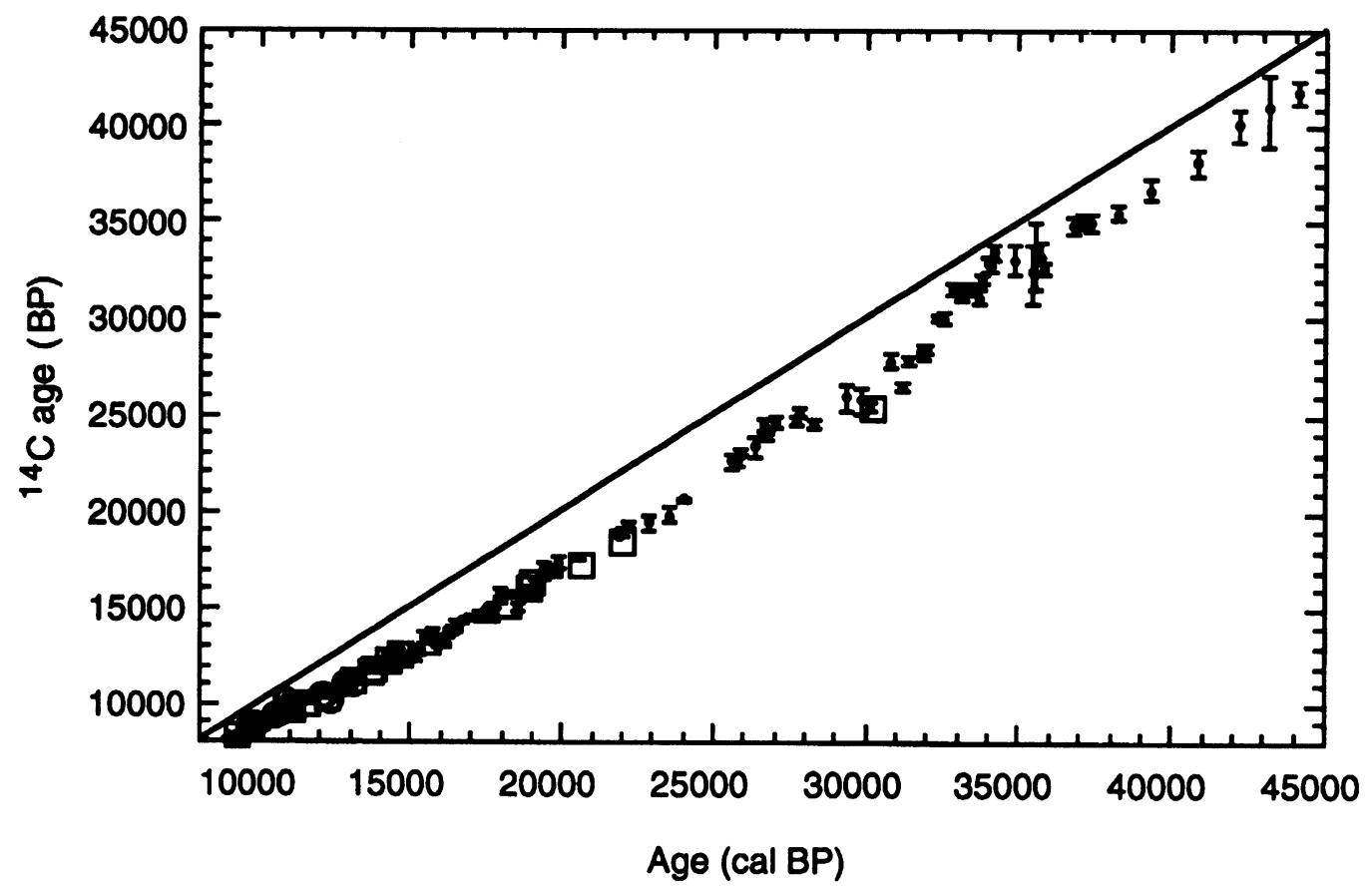

Fig. 3. Atmospheric radiocarbon calibration for the complete ${ }^{14} \mathrm{C}$ dating range ( $<45 \mathrm{ka} \mathrm{cal} \mathrm{BP}$ ) reconstructed from annually laminated sediments from Lake Suigetsu (Japan). $\bullet$ with 1- $\sigma$ bars = Lake Suigetsu. $\Delta$ (Mururoa: Bard et al. 1993), $\square$ (Barbados: Bard et al. 1993), and O (Papua New Guinea: Edwards et al 1993) correspond to U-series based ${ }^{14} \mathrm{C}$ calibration on corals. The numbers of varve and ${ }^{14} \mathrm{C}$ ages of Lake Suigetsu are listed in the Appendix.

matic changes, as well as for better understanding of geophysical changes in the Earth's system. A detailed discussion of our results from this point of view will be published elsewhere (Kitagawa and van der Plicht 1998).

The long sequence of annually laminated sediments from Lake Suigetsu provides a very exciting record of atmospheric ${ }^{14} \mathrm{C}$ changes during the past $45 \mathrm{ka}$. In order to produce a more complete ${ }^{14} \mathrm{C}$ calibration curve, we intend to completely reconstruct the continuous varve chronology for this period together with other paleoenvironmental signals recorded in these sediments.

\section{ACKNOWLEDGMENTS}

The unpublished data on Lake Gościąz varve chronology were kindly provided by Tomasz Goslar of the Institute of Physics, Silesian Technical University, Gliwice, Poland. This work was sponsored partly by the Grant-Aid for Scientific Research from the Ministry of Education, Science and Culture (no. 04212116) and by the Toyota Foundation (96-A-232). 


\section{REFERENCES}

Bard, E., Arnold, M., Fairbanks, R. G. and Hamelin, B. $1993{ }^{230} \mathrm{Th}-{ }^{234} \mathrm{U}$ and ${ }^{14} \mathrm{C}$ ages obtained by mass spectrometry on corals. In Stuiver, M., Long, A. and Kra, R. S., eds., Calibration 1993. Radiocarbon 35(1): 191-199.

Bard, E., Hamelin, B., Fairbanks, R. G. and Zindler, A. 1990 Calibration of the ${ }^{14} \mathrm{C}$ timescale over the past 30,000 years using mass spectrometric U-Th ages from Barbados Corals. Nature 345: 405-410.

Björck, S., Kromer, B., Johnsen, S., Bennike, O., Hammarlund, D., Lemdahl, G., Possnert, G., Rasmussen, T. L., Wohlfarth, B., Hammer, C. U. and Spurk, M. 1996 Synchronised terrestrial-atmospheric deglacial records around the North Atlantic. Science 274: 11551160.

Edwards, R. L., Beck, J. W., Burr, G. S., Donahue, D. L., Chappell, J. M. A., Bloom, A. L., Druffel, E. R. M. and Taylor, F. W. 1993 A large drop in atmospheric ${ }^{14} \mathrm{C} /$ ${ }^{12} \mathrm{C}$ and reduced melting in Younger Dryas, documented with ${ }^{230} \mathrm{Th}$ ages of corals. Science 260 : 962967.

Goslar, T., Arnold, M., Bard, E., Kuc, T., Pazdur, M. F., Ralska-Jasiewiczowa, M., Rózanski, K., Tisnerat, N., Walanus, A., Wicik, B. and Wieckowski, K. 1995 High concentration of atmospheric ${ }^{14} \mathrm{C}$ during the Younger Dryas cold episode. Nature 377: 414-417.

Goslar, T., Arnold, M. and Tisnerat-Laborde, N. (ms.) An updated synchronization of the Lake Gosciaz varve chronology with the German pine and oak chronologies. In preparation.

Gottdang, A., Mous, D. J. W. and van der Plicht, J. 1995 The HVEE ${ }^{14} \mathrm{C}$ system at Groningen. In Cook, G. T., Harkness, D. D., Miller, B. F. and Scott, E. M., eds., Proceedings of the 15 th International ${ }^{14} \mathrm{C}$ Conference. Radiocarbon 37(2): 649-656.

Kitagawa, H., Fukusawa, H., Nakamura, T., Okamura, M., Takemura, K., Hayashida, A. and Yasuda, Y. 1995 AMS ${ }^{14} \mathrm{C}$ dating of varved sediments from Lake Suigetsu, central Japan and atmospheric ${ }^{14} \mathrm{C}$ change during the late Pleistocene. In Cook, G. T., Harkness, D. D., Miller, B. F. and Scott, E. M., eds., Proceedings of the 15 th International ${ }^{14} \mathrm{C}$ Conference. Radiocarbon 37(2): 371-378.

Kitagawa, H. and van der Plicht, J. 1997 Enrichment of sub-milligram size carbon samples. Nuclear Instruments and Methods in Physics Research B123: 218220.

1998 Atmospheric radiocarbon for the complete ${ }^{14} \mathrm{C}$ dating range: Late glacial fluctuations and cosmogenic isotope production. Science 279: 1187-1190.

Kromer, B., Ambers, J., Baillie, M. G. L., Damon, P. E., Hesshaimer, V., Hofmann, J., Joris, O., Levin, I., Manning, W., McCormac, F. G., van der Plicht, J., Spurk, M., Stuiver, M. and Weninger, B. 1996 Report: Summary of the workshop "Aspects of high-precision radiocarbon calibration". Radiocarbon 38(3): 607-610.

Kromer, B. and Becker, B. 1993 German oak and pine ${ }^{14} \mathrm{C}$ calibration, 7200-9439 BC. In Stuiver, M., Long, A. and Kra, R. S., eds., Calibration 1993. Radiocarbon 35(1): 125-135.

Laj, C., Mazaud, A. and Duplessy, J.-C. 1996 Geomagnetic intensity and ${ }^{14} \mathrm{C}$ abundance in the atmosphere and ocean during the past $50 \mathrm{kyr}$. Geophysical Research Letters 23(16): 2045-2048.

Mook, W. G. and Streurman, H. J. 1983 Physical and chemical aspects of radiocarbon dating. In Mook, W. G. and Waterbolk, H. T., eds., Proceedings of the 1st International Symposium, ${ }^{14} \mathrm{C}$ and Archaeology. PACT 8. Strasbourg: Conseil de l'Europe, Assemblée parlementaire: $31-55$.

Takemura, K., Kitagawa, H., Hayashida, A. and Yasuda, Y. 1994 Sedimentary facies and chronology of core samples from Lake Mikata, Lake Suigetsu and Kurota Lowland, central Japan - sedimentary environment in Mikata Lowland since the last interglacial time. Journal of Geography 103(3): 233-242.

van der Plicht, J., Aerts, A., Wijma, S. and Zondervan, A. 1995 First Results from the Groningen AMS facility. In Cook, G. T., Harkness, D. D., Miller, B. F. and Scott, E. M., eds., Proceedings of the 15th International ${ }^{14} \mathrm{C}$ Conference. Radiocarbon 37(2): 657-661.

Voelker, A., Sarnthein, H., Grootes, P., Erlenkeuser, H., Laj, C., Mazaud, A., Nadeau, M.-J., and Schleicher, M. 1998 Correlation of marine ${ }^{14} \mathrm{C}$ ages from the Nordic Seas with the GISP2 isotope record: Implications for ${ }^{14} \mathrm{C}$ calibration beyond $25 \mathrm{ka} \mathrm{BP}$. Radiocarbon, this issue.

Wijma, S. and van der Plicht, J. 1997 The Groningen AMS tandetron. Nuclear Instruments and Methods in Physics Research B123: 218-220.

Wohlfarth, B., Björck, S., Possnert, G., Lemdahl, G., Brunnberg, L., Ising, L., Olsson, S. and Svensson, N.-O. 1993 AMS dating Swedish varved clays of the last glacial/interglacial transition and the potential difficulties of calibrating Late Weichselian 'absolute' chronologies. Boreas 22: 113-128. 


\section{APPENDIX}

Varve and ${ }^{14} \mathrm{C}$ chronologies of the annually laminated sediments from the Lake Suigetsu, Japan. The numbers in the FVT column represent relative varve numbers from the beginning of the presently floating varve chronology $(1042.0 \mathrm{~cm}$ below the top sediment). The varve age below $3045.0 \mathrm{~cm}$ is estimated by assuming a constant sedimentation $\left(0.62 \mathrm{~mm} \mathrm{yr}^{-1}\right)$.

\begin{tabular}{|c|c|c|c|c|c|}
\hline \multirow[b]{2}{*}{ Sample } & \multirow{2}{*}{$\begin{array}{l}\text { Depth in SG } \\
\quad(\mathrm{cm})\end{array}$} & \multicolumn{2}{|c|}{ Varve age } & \multirow{2}{*}{$\begin{array}{l}\text { Lab code(s) } \\
\text { GrA- }\end{array}$} & \multirow{2}{*}{$\begin{array}{l}{ }^{14} \mathrm{C} \text { age } \\
\text { (yr BP) }\end{array}$} \\
\hline & & FVT & cal BP & & \\
\hline SG02C04 & $111.7 \pm 1.5$ & & & 4571 & $525 \pm 60$ \\
\hline SG02A02 & $146.4 \pm 1.5$ & & & 4572 & $940 \pm 65$ \\
\hline SG03E05 & $177.1 \pm 2.0$ & & & 4573 & $1045 \pm 60$ \\
\hline SG03A03 & $240.4 \pm 1.5$ & & & 4574 & $1575 \pm 60$ \\
\hline SG04D05 & $291.7 \pm 1.5$ & & & 4575 & $2140 \pm 90$ \\
\hline SG04A01 & $328.4 \pm 1.5$ & & & 4576 & $2285 \pm 65$ \\
\hline SG05D07 & $382.7 \pm 1.5$ & & & 4577 & $2470 \pm 60$ \\
\hline SG05C01 & $385.5 \pm 1.5$ & & & 4578 & $2450 \pm 65$ \\
\hline SG05B05 & $410.6 \pm 1.5$ & & & 4579 & $2590 \pm 65$ \\
\hline SG06E05 & $452.9 \pm 2.2$ & & & 4580 & $3040 \pm 60$ \\
\hline SG06C03 & $479.8 \pm 1.5$ & & & 4581 & $3115 \pm 60$ \\
\hline SG06B07 & $515.4 \pm 1.5$ & & & 4582 & $3420 \pm 60$ \\
\hline SG07E08 & $562.7 \pm 2.0$ & & & 1899 & $3680 \pm 60$ \\
\hline SG07E08 & $569.2 \pm 1.5$ & & & 1897 & $3705 \pm 60$ \\
\hline SG07B05 & $604.9 \pm 1.5$ & & & 1900 & $3870 \pm 60$ \\
\hline SG08C02 & $626.6 \pm 1.5$ & & & 1953 & $4120 \pm 60$ \\
\hline SG08C10 & $652.1 \pm 1.5$ & & & 7746 & $4416 \pm 40$ \\
\hline SG08B08 & $677.5 \pm 1.5$ & & & 1939 & $4460 \pm 60$ \\
\hline SG08A03 & $692.5 \pm 1.5$ & & & 1894 & $4525 \pm 60$ \\
\hline SG09D04 & $728.4 \pm 1.5$ & & & 2493 & $4725 \pm 60$ \\
\hline SG09C01 & $748.1 \pm 1.5$ & & & 2498 & $4875 \pm 60$ \\
\hline SG09C05 & $760.0 \pm 1.5$ & & & 4583 & $5095 \pm 125$ \\
\hline SG09B01 & $775.8 \pm 1.5$ & & & 4584 & $5040 \pm 70$ \\
\hline SG10C05 & $817.0 \pm 1.5$ & & & 2499 & $5460 \pm 60$ \\
\hline SG10C09 & $829.4 \pm 1.5$ & & & 2496 & $5420 \pm 65$ \\
\hline SG10B03 & $844.9 \pm 1.5$ & & & 2495 & $5435 \pm 65$ \\
\hline SG10B05 & $851.1 \pm 1.5$ & & & 2500 & $5800 \pm 85$ \\
\hline SG10B07 & $857.3 \pm 1.5$ & & & 2494 & $5775 \pm 70$ \\
\hline SG10B11 & $869.7 \pm 1.5$ & & & 2491 & $5850 \pm 70$ \\
\hline SG10A02 & $875.9 \pm 1.5$ & & & 7744 & $5960 \pm 60$ \\
\hline SG10A05 & $885.2 \pm 1.5$ & & & 7742 & $5890 \pm 60$ \\
\hline SG10A08 & $894.0 \pm 1.5$ & & & 7745 & $6250 \pm 90$ \\
\hline SG11D02 & $912.8 \pm 1.5$ & & & 2963 & $6390 \pm 200$ \\
\hline SG11D03 & $915.8 \pm 1.5$ & & & 7738 & $6220 \pm 70$ \\
\hline SG11D04 & $919.0 \pm 1.5$ & & & 2964 & $6455 \pm 110$ \\
\hline SG11B03 & $925.2 \pm 1.5$ & & & 2965 & $6445 \pm 100$ \\
\hline SG11B03 & $931.9 \pm 2.0$ & & & 2966 & $6675 \pm 150$ \\
\hline SG11D10 & $937.8 \pm 1.3$ & & & 2967 & $6585 \pm 150$ \\
\hline SG11C01 & $940.7 \pm 1.5$ & & & 7741 & $6410 \pm 80$ \\
\hline SG11C02 & $943.8 \pm 1.5$ & & & 2982 & $6520 \pm 115$ \\
\hline SG11C03 & $946.9 \pm 1.5$ & & & 7734 & $6500 \pm 70$ \\
\hline SG11C04 & $950.0 \pm 1.5$ & & & 2977 & $6590 \pm 95$ \\
\hline
\end{tabular}




\begin{tabular}{|c|c|c|c|c|c|}
\hline \multirow[b]{2}{*}{ Sample } & \multirow{2}{*}{$\begin{array}{l}\text { Depth in SG } \\
(\mathrm{cm})\end{array}$} & \multicolumn{2}{|c|}{ Varve age } & \multirow{2}{*}{$\begin{array}{c}\text { Lab code(s) } \\
\text { GrA- }\end{array}$} & \multirow{2}{*}{$\begin{array}{l}{ }^{14} \mathrm{C} \text { age } \\
\text { (yr BP) }\end{array}$} \\
\hline & & FVT & cal BP & & \\
\hline SG11C06 & $956.4 \pm 1.8$ & & & 2989 & $6635 \pm 110$ \\
\hline SG11B01 & $959.8 \pm 1.5$ & & & 7733 & $6780 \pm 90$ \\
\hline SG11B02 & $962.9 \pm 1.5$ & & & 2978 & $6670 \pm 85$ \\
\hline SG11B03 & $966.0 \pm 1.5$ & & & 7732 & $6770 \pm 70$ \\
\hline SG11B04 & $969.1 \pm 1.5$ & & & 7730 & $6930 \pm 70$ \\
\hline SG11B05 & $972.2 \pm 1.5$ & & & 2981 & $7330 \pm 200$ \\
\hline SG11B06 & $976.1 \pm 2.3$ & & & 7743 & $6990 \pm 60$ \\
\hline SG11A01 & $979.1 \pm 1.5$ & & & 7731 & $6850 \pm 70$ \\
\hline SG11A03 & $986.1 \pm 1.5$ & & & 7737 & $7030 \pm 70$ \\
\hline SG11A06 & $995.4 \pm 1.5$ & & & 7736 & $6920 \pm 70$ \\
\hline SG12C01 & $988.6 \pm 1.5$ & & & 2930 & $7530 \pm 100$ \\
\hline SG12C05 & $1001.0 \pm 1.5$ & & & 2846 & $7445 \pm 100$ \\
\hline SG12B07 & $1009.3 \pm 1.5$ & & & 6231 & $7315 \pm 85$ \\
\hline SG12B04 & $1021.7 \pm 1.5$ & & & 2888,2889 & $7500 \pm 60$ \\
\hline SG12B06 & $1025.3 \pm 2.0$ & & & 2946 & $7480 \pm 100$ \\
\hline SG12A01 & $1028.9 \pm 1.5$ & & & 6230 & $7325 \pm 110$ \\
\hline SG13D01 & $1043.6 \pm 1.5$ & 13 & 8841 & 6234 & $7610 \pm 70$ \\
\hline SG13D04 & $1053.4 \pm 1.5$ & 91 & 8919 & 2849 & $7805 \pm 100$ \\
\hline SG13D07 & $1063.1 \pm 1.5$ & 172 & 9000 & 6233 & $8020 \pm 90$ \\
\hline SG13D08 & $1066.4 \pm 1.5$ & 200 & 9028 & 6232 & $8035 \pm 85$ \\
\hline SG13D09 & $1070.2 \pm 2.0$ & 231 & 9059 & 2839 & $8150 \pm 105$ \\
\hline SG13C03 & $1079.4 \pm 1.5$ & 301 & 9129 & 2914 & $8020 \pm 105$ \\
\hline SG13C05 & $1085.9 \pm 1.5$ & 343 & 9171 & 6235 & $8050 \pm 80$ \\
\hline SG13C06 & $1089.1 \pm 1.5$ & 368 & 9196 & 6236 & $8035 \pm 95$ \\
\hline SG13C07 & $1092.4 \pm 1.5$ & 393 & 9221 & 2840 & $8085 \pm 85$ \\
\hline SG13C09 & $1097.5 \pm 1.3$ & 413 & 9241 & 2947,2948 & $8050 \pm 70$ \\
\hline SG13B05 & $1114.3 \pm 2.3$ & 553 & 9381 & 2901 & $8200 \pm 105$ \\
\hline SG13A04 & $1128.1 \pm 1.5$ & 663 & 9491 & 2842 & $8635 \pm 110$ \\
\hline SG14D03 & $1142.0 \pm 1.5$ & 750 & 9578 & 2843 & $8635 \pm 110$ \\
\hline SG14D06 & $1151.7 \pm 1.5$ & 821 & 9649 & 3087 & $8765 \pm 80$ \\
\hline SG14C01 & $1159.2 \pm 1.5$ & 878 & 9706 & 2835 & $8775 \pm 110$ \\
\hline SG14C04 & $1168.8 \pm 1.5$ & 950 & 9778 & 3085 & $8900 \pm 90$ \\
\hline SG14C06 & $1175.2 \pm 1.5$ & 998 & 9826 & 3080 & $9055 \pm 90$ \\
\hline SG14B02 & $1184.3 \pm 1.5$ & 1067 & 9895 & 2844 & $8845 \pm 110$ \\
\hline SG14B07 & $1200.4 \pm 1.5$ & 1204 & 10,032 & 3082 & $8830 \pm 95$ \\
\hline SG14A04 & $1213.2 \pm 1.5$ & 1298 & 10,126 & 2890 & $8665 \pm 110$ \\
\hline SG15D01 & $1226.7 \pm 1.5$ & 1385 & 10,213 & 3079 & $8970 \pm 120$ \\
\hline SG15D05 & $1240.2 \pm 1.5$ & 1485 & 10,313 & 2971 & $9280 \pm 115$ \\
\hline SG15D07 & $1247.0 \pm 1.5$ & 1555 & 10,383 & 2845 & $9150 \pm 115$ \\
\hline SG15C01 & $1250.4 \pm 1.5$ & 1575 & 10,403 & 2921 & $9270 \pm 115$ \\
\hline SG15C03 & $1257.2 \pm 1.5$ & 1625 & 10,453 & 4585 & $9260 \pm 180$ \\
\hline SG15C06 & $1267.3 \pm 1.5$ & 1685 & 10,513 & 2915 & $9635 \pm 100$ \\
\hline SG15C08 & $1274.1 \pm 1.5$ & 1735 & 10,563 & 3081 & $9535 \pm 80$ \\
\hline SG15B02 & $1280.9 \pm 1.5$ & 1775 & 10,603 & 2847 & $9525 \pm 90$ \\
\hline SG15B03 & $1284.3 \pm 1.5$ & 1805 & 10,633 & 2944 & $9320 \pm 90$ \\
\hline SG15B06 & $1294.4 \pm 1.5$ & 1885 & 10,713 & 2913 & $9405 \pm 80$ \\
\hline SG15B07 & $1297.8 \pm 1.5$ & 1915 & 10,743 & 2912 & $9625 \pm 100$ \\
\hline SG15A01 & $1305.1 \pm 1.5$ & 1970 & 10,798 & 3083 & $9555 \pm 105$ \\
\hline SG15A04 & $1315.3 \pm 1.5$ & 2045 & 10,873 & 2907 & $9495 \pm 90$ \\
\hline
\end{tabular}




\begin{tabular}{|c|c|c|c|c|c|}
\hline \multirow[b]{2}{*}{ Sample } & \multirow{2}{*}{$\begin{array}{l}\text { Depth in SG } \\
\quad(\mathrm{cm})\end{array}$} & \multicolumn{2}{|c|}{ Varve age } & \multirow{2}{*}{$\begin{array}{c}\text { Lab code(s) } \\
\text { GrA- }\end{array}$} & \multirow{2}{*}{$\begin{array}{l}{ }^{14} \mathrm{C} \text { age } \\
\text { (yr BP) }\end{array}$} \\
\hline & & FVT & cal BP & & \\
\hline SG16D06 & $1336.7 \pm 2.0$ & 2296 & 11,124 & 3086 & $10,075 \pm 85$ \\
\hline SG16C01 & $1344.2 \pm 1.5$ & 2371 & 11,199 & 2904 & $10,005 \pm 95$ \\
\hline SG16C04 & $1254.2 \pm 1.5$ & 2453 & 11,281 & 2905 & $9860 \pm 95$ \\
\hline SG16C05 & $1357.8 \pm 1.8$ & 2492 & 11,320 & 2911 & $10,125 \pm 95$ \\
\hline SG16B01 & $1364.2 \pm 1.5$ & 2543 & 11,371 & 2961 & $10,095 \pm 125$ \\
\hline SG16B02 & $1367.5 \pm 1.5$ & 2572 & 11,400 & 2838 & $10,055 \pm 100$ \\
\hline SG16B04 & $1374.2 \pm 1.5$ & 2633 & 11,461 & 2917 & $10,145 \pm 95$ \\
\hline SG16B05 & $1377.5 \pm 1.5$ & 2663 & 11,491 & 2916 & $10,095 \pm 100$ \\
\hline SG16A02 & $1386.9 \pm 1.5$ & 2752 & 11,580 & 3078 & $10,285 \pm 85$ \\
\hline SG16A05 & $1393.6 \pm 1.5$ & 2814 & 11,642 & 2902 & $10,165 \pm 95$ \\
\hline SG16A06 & $1396.9 \pm 1.5$ & 2854 & 11,682 & 2909 & $10,115 \pm 95$ \\
\hline SG17D01 & $1410.5 \pm 1.5$ & 2981 & 11,809 & 2969 & $10,100 \pm 105$ \\
\hline SG17D02 & $1413.5 \pm 1.5$ & 3022 & 11,850 & 2836 & $10,455 \pm 100$ \\
\hline SG17D06 & $1425.6 \pm 1.5$ & 3177 & 12,005 & 2970 & $10,395 \pm 105$ \\
\hline SG17D10 & $1437.4 \pm 1.3$ & 3315 & 12,143 & 2981 & $10,370 \pm 125$ \\
\hline SG17C03 & $1446.2 \pm 1.5$ & 3433 & 12,261 & 2837 & $10,710 \pm 110$ \\
\hline SG17C04 & $1449.2 \pm 1.5$ & 3474 & 12,302 & 2913 & $10,590 \pm 95$ \\
\hline SG17B01 & $1455.3 \pm 1.5$ & 3540 & 12,368 & 2906 & $10,380 \pm 90$ \\
\hline SG17B04 & $1464.3 \pm 1.5$ & 3652 & 12,480 & 2848 & $10,670 \pm 100$ \\
\hline SG17A02 & $1483.9 \pm 1.5$ & 3908 & 12,736 & 2908 & $10,700 \pm 100$ \\
\hline SG17A04 & $1490.0 \pm 1.5$ & 3965 & 12,793 & 2920 & $10,915 \pm 125$ \\
\hline SG17A07 & $1497.2 \pm 0.8$ & 4030 & 12,858 & 3077 & $11,000 \pm 125$ \\
\hline SG18E01 & $1499.0 \pm 1.5$ & 4046 & 12,874 & 4532 & $11,030 \pm 55$ \\
\hline SG18E02 & $1502.0 \pm 1.5$ & 4096 & 12,924 & 5634 & $11,415 \pm 145$ \\
\hline SG18E03 & $1505.0 \pm 1.5$ & 4136 & 12,964 & 5635 & $11,210 \pm 90$ \\
\hline SG18E04 & $1508.0 \pm 1.5$ & 4176 & 13,004 & 5637 & $11,335 \pm 90$ \\
\hline SG18E05 & $1511.0 \pm 1.5$ & 4216 & 13,044 & 4533 & $10,975 \pm 55$ \\
\hline SG18E06 & $1514.0 \pm 1.5$ & 4256 & 13,084 & 5638 & $11,440 \pm 110$ \\
\hline SG18E07 & $1517.0 \pm 1.5$ & 4296 & 13,124 & 5639 & $11,480 \pm 85$ \\
\hline SG18D01 & $1522.5 \pm 1.5$ & 4376 & 13,204 & 4534 & $11,460 \pm 55$ \\
\hline SG18D02 & $1525.5 \pm 1.5$ & 4426 & 13,254 & 5640 & $11,690 \pm 85$ \\
\hline SG18C01 & $1540.5 \pm 1.5$ & 4686 & 13,514 & 5641 & $11,830 \pm 65$ \\
\hline SG18C04 & $1548.5 \pm 1.5$ & 4816 & 13,644 & 4535 & $12,000 \pm 330$ \\
\hline SG18B01 & $1554.8 \pm 1.8$ & 4906 & 13,734 & 5653 & $11,980 \pm 110$ \\
\hline SG18B03 & $1561.0 \pm 1.5$ & 5006 & 13,834 & 4536 & $12,040 \pm 55$ \\
\hline SG18B05 & $1567.0 \pm 1.5$ & 5106 & 13,934 & 6206 & $12,245 \pm 125$ \\
\hline SG18B06 & $1570.3 \pm 1.8$ & 5166 & 13,994 & 4537 & $12,050 \pm 85$ \\
\hline SG18A01 & $1573.5 \pm 1.5$ & 5216 & 14,044 & 5642 & $12,250 \pm 95$ \\
\hline SG18A04 & $1582.5 \pm 1.5$ & 5346 & 14,174 & 6202 & $12,270 \pm 95$ \\
\hline SG18A06 & $1588.0 \pm 1.5$ & 5426 & 14,254 & 5654 & $12,610 \pm 295$ \\
\hline SG19D03 & $1594.8 \pm 1.5$ & 5531 & 14,359 & 4539 & $12,260 \pm 60$ \\
\hline SG19D04 & $1598.0 \pm 1.5$ & 5591 & 14,419 & 6204 & $12,410 \pm 100$ \\
\hline SG19D05 & $1601.3 \pm 1.5$ & 5621 & 14,449 & 5643 & $12,425 \pm 85$ \\
\hline SG19D07 & $1607.7 \pm 1.5$ & 5721 & 14,549 & 4540 & $12,320 \pm 55$ \\
\hline SG19D08 & $1610.9 \pm 1.5$ & 5771 & 14,599 & 5644 & $12,680 \pm 150$ \\
\hline SG19C04 & $1623.8 \pm 1.5$ & 5961 & 14,789 & 5645 & $12,520 \pm 70$ \\
\hline SG19C05 & $1627.0 \pm 1.5$ & 6031 & 14,859 & 4541 & $12,345 \pm 55$ \\
\hline SG19B01 & $1638.8 \pm 1.5$ & 6171 & 14,999 & 4542 & $12,490 \pm 55$ \\
\hline SG19B04 & $1648.4 \pm 1.5$ & 6331 & 15,159 & 5646 & $12,625 \pm 370$ \\
\hline
\end{tabular}




\begin{tabular}{|c|c|c|c|c|c|}
\hline \multirow[b]{2}{*}{ Sample } & \multirow{2}{*}{$\begin{array}{l}\text { Depth in SG } \\
\quad(\mathrm{cm})\end{array}$} & \multicolumn{2}{|c|}{ Varve age } & \multirow{2}{*}{$\begin{array}{l}\text { Lab code(s) } \\
\text { GrA- }\end{array}$} & \multirow{2}{*}{$\begin{array}{l}{ }^{14} \mathrm{C} \text { age } \\
\text { (yr BP) }\end{array}$} \\
\hline & & FVT & cal BP & & \\
\hline SG19B05 & $1651.6 \pm 1.5$ & 6389 & 15,217 & 4543 & $12,745 \pm 75$ \\
\hline SG19B06 & $1654.8 \pm 1.5$ & 6441 & 15,269 & 6205 & $12,705 \pm 105$ \\
\hline SG19A03 & $1668.8 \pm 1.5$ & 6681 & 15,509 & 5648 & $13,440 \pm 300$ \\
\hline SG20D01 & $1684.7 \pm 1.5$ & 6966 & 15,794 & 4550 & $13,665 \pm 215$ \\
\hline SG20D03 & $1691.0 \pm 1.5$ & 7066 & 15,894 & 5649 & $13,385 \pm 165$ \\
\hline SG20D04 & $1694.1 \pm 1.5$ & 7116 & 15,944 & 4551 & $13,015 \pm 80$ \\
\hline SG20D05 & $1697.3 \pm 1.5$ & 7176 & 16,004 & 5650 & $13,475 \pm 100$ \\
\hline SG20C01 & $1706.7 \pm 1.5$ & 7326 & 16,154 & 5636 & $13,105 \pm 110$ \\
\hline $\mathrm{SG} 20 \mathrm{C} 03$ & $1712.9 \pm 1.5$ & 7416 & 16,244 & 4552 & $13,572 \pm 60$ \\
\hline SG20C05 & $1719.2 \pm 1.5$ & 7526 & 16,354 & 5651 & $13,885 \pm 80$ \\
\hline SG20C06 & $1722.4 \pm 1.5$ & 7586 & 16,414 & 4553 & $13,855 \pm 125$ \\
\hline SG20B01 & $1729.2 \pm 1.5$ & 7706 & 16,534 & 6203 & $14,205 \pm 170$ \\
\hline SG20B02 & $1732.3 \pm 1.5$ & 7746 & 16,574 & 4554 & $13,815 \pm 70$ \\
\hline SG20B04 & $1738.6 \pm 1.5$ & 7856 & 16,684 & 5652 & $14,295 \pm 85$ \\
\hline SG20A03 & $1757.4 \pm 1.5$ & 8136 & 16,964 & 4555 & $14,440 \pm 95$ \\
\hline SG21D04 & $1778.6 \pm 1.5$ & 8466 & 17,294 & 4556 & $14,695 \pm 60$ \\
\hline SG21D07 & $1787.7 \pm 1.5$ & 8616 & 17,444 & 4557 & $14,595 \pm 90$ \\
\hline SG21C02 & $1795.8 \pm 1.5$ & 8736 & 17,564 & 4558 & $14,630 \pm 60$ \\
\hline $\mathrm{SG} 21 \mathrm{C} 02$ & $1801.9 \pm 1.5$ & 8841 & 17,669 & 4559 & $14,860 \pm 195$ \\
\hline SG21C07 & $1811.0 \pm 1.5$ & 8991 & 17,819 & 4556 & $15,125 \pm 185$ \\
\hline SG21B03 & $1820.1 \pm 1.5$ & 9131 & 17,959 & 4561 & $15,755 \pm 270$ \\
\hline SG21B03 & $1823.1 \pm 1.5$ & 9181 & 18,009 & 5658 & $15,480 \pm 140$ \\
\hline SG21B03 & $1826.2 \pm 1.5$ & 9232 & 18,060 & 5668 & $15,730 \pm 145$ \\
\hline SG21A05 & $1847.9 \pm 2.0$ & 9616 & 18,444 & 4562 & $15,695 \pm 180$ \\
\hline SG22D03 & $1864.1 \pm 2.0$ & 9886 & 18,714 & 4564 & $15,915 \pm 230$ \\
\hline SG22D06 & $1874.0 \pm 1.3$ & 10,011 & 18,839 & 4565 & $15,990 \pm 180$ \\
\hline SG22C06 & $1891.9 \pm 2.5$ & 10,296 & 19,124 & 4566 & $16,280 \pm 195$ \\
\hline SG22B02 & $1901.0 \pm 1.5$ & 10,466 & 19,294 & 5669 & $16,750 \pm 220$ \\
\hline SG22B04 & $1907.1 \pm 1.5$ & 10,571 & 19,399 & 5668 & $16,700 \pm 178$ \\
\hline SG22B05 & $1910.7 \pm 2.0$ & 10,636 & 19,464 & 4567 & $17,065 \pm 240$ \\
\hline SG22A01 & $1919.3 \pm 1.5$ & 10,791 & 19,619 & 4586 & $17,135 \pm 170$ \\
\hline SG22A04 & $1928.4 \pm 1.5$ & 10,971 & 19,799 & 4569 & $16,950 \pm 185$ \\
\hline SG22A05 & $1931.4 \pm 1.5$ & 11,023 & 19,851 & 4570 & $16,760 \pm 185$ \\
\hline SG22A06 & $1934.4 \pm 1.5$ & 11,081 & 19,909 & 5660 & $17,380 \pm 240$ \\
\hline SG23-4 & $1968.7 \pm 0.5$ & 11,716 & 20,544 & 6193 & $17,745 \pm 140$ \\
\hline SG24-5 & $2051.1 \pm 0.5$ & 13,117 & 21,945 & 6192 & $18,810 \pm 110$ \\
\hline SG24-4 & $2054.3 \pm 0.5$ & 13,172 & 22,000 & 6191 & $18,975 \pm 290$ \\
\hline SG24-3 & $2065.4 \pm 0.5$ & 13,372 & 22,200 & 6190 & $19,370 \pm 135$ \\
\hline SG24-1 & $2106.9 \pm 0.5$ & 14,032 & 22,860 & 6189 & $19,425 \pm 305$ \\
\hline SG25-2 & $2149.5 \pm 0.5$ & 14,752 & 23,580 & 6188 & $19,830 \pm 365$ \\
\hline SG25-1 & $2175.5 \pm 0.5$ & 15,182 & 24,010 & 6187 & $20,630 \pm 130$ \\
\hline SG26-3 & $2264.0 \pm 2.0$ & 16,732 & 25,560 & 6186 & $22,600 \pm 440$ \\
\hline SG26-2 & $2278.4 \pm 0.5$ & 16,957 & 25,785 & 6185 & $22,630 \pm 220$ \\
\hline SG26-1 & $2286.1 \pm 0.5$ & 17,067 & 25,895 & 6184 & $23,170 \pm 150$ \\
\hline SG27-7 & $2312.2 \pm 0.5$ & 17,492 & 26,320 & 6183 & $23,400 \pm 500$ \\
\hline SG27-5 & $2334.7 \pm 0.5$ & 17,852 & 26,680 & 6182 & $24,495 \pm 270$ \\
\hline SG27-4 & $2337.1 \pm 0.5$ & 17,902 & 26,730 & 6181 & $23,890 \pm 210$ \\
\hline SG27-3 & $2340.4 \pm 0.5$ & 17,957 & 26,785 & 6180 & $23,970 \pm 170$ \\
\hline SG27-2 & $2356.2 \pm 0.5$ & 18,206 & 27,034 & 6179 & $24,595 \pm 265$ \\
\hline
\end{tabular}




\begin{tabular}{|c|c|c|c|c|c|}
\hline \multirow[b]{2}{*}{ Sample } & \multirow{2}{*}{$\begin{array}{l}\text { Depth in SG } \\
\quad(\mathrm{cm})\end{array}$} & \multicolumn{2}{|c|}{ Varve age } & \multirow{2}{*}{$\begin{array}{l}\text { Lab code(s) } \\
\text { GrA- }\end{array}$} & \multirow{2}{*}{$\begin{array}{c}{ }^{14} \mathrm{C} \text { age } \\
\text { (yr BP) }\end{array}$} \\
\hline & & FVT & cal BP & & \\
\hline SG28-4 & $2405.8 \pm 0.5$ & 18,902 & 27,730 & 6178 & $24,700 \pm 270$ \\
\hline SG28-3 & $2408.4 \pm 0.5$ & 18,982 & 27,810 & 6177 & $25,130 \pm 185$ \\
\hline SG28-2 & $2433.0 \pm 0.5$ & 19,422 & 28,250 & 6176 & $24,545 \pm 270$ \\
\hline SG29-3 & $2508.2 \pm 0.5$ & 20,487 & 29,315 & 6173 & $25,980 \pm 670$ \\
\hline SG29-2 & $2537.6 \pm 0.5$ & 21,002 & 29,830 & 6172 & $25,840 \pm 670$ \\
\hline SG29-1 & $2560.8 \pm 0.5$ & 21,312 & 30,140 & 6171 & $25,445 \pm 190$ \\
\hline SG30-5 & $2623.4 \pm 0.5$ & 22,342 & 31,170 & 6168 & $26,460 \pm 215$ \\
\hline SG30-4 & $2636.4 \pm 0.5$ & 22,572 & 31,400 & 6169 & $27,880 \pm 235$ \\
\hline SG30R-1 & $2665.5 \pm 0.5$ & 23,012 & 31,840 & 6174 & $28,495 \pm 250$ \\
\hline SG30-3 & $2665.5 \pm 0.5$ & 23,012 & 31,840 & 6170 & $28,220 \pm 245$ \\
\hline SG30-1 & $2671.8 \pm 0.5$ & 23,092 & 31,920 & 6167 & $28,495 \pm 255$ \\
\hline SG31-7 & $2698.3 \pm 0.5$ & 23,472 & 32,300 & 5618,6200 & $30,080 \pm 200$ \\
\hline SG31-6 & $2716.5 \pm 0.5$ & 23,757 & 32,585 & 5617 & $30,010 \pm 310$ \\
\hline SG31-5 & $2733.1 \pm 0.5$ & 23,997 & 32,825 & 5616 & $31,545 \pm 340$ \\
\hline SG31-4 & $2740.0 \pm 0.5$ & 24,122 & 32,950 & 5615 & $31,545 \pm 335$ \\
\hline SG31-1 & $2751.8 \pm 0.5$ & 24,312 & 33,140 & 5613 & $31,345 \pm 355$ \\
\hline SG31-3 & $2761.4 \pm 0.5$ & 24,472 & 33,300 & 5614 & $31,547 \pm 330$ \\
\hline SG31-7 & $2784.8 \pm 0.5$ & 24,877 & 33,705 & 5625 & $31,190 \pm 360$ \\
\hline SG32-6 & $2792.2 \pm 0.5$ & 25,007 & 33,835 & 5624,6199 & $32,140 \pm 260$ \\
\hline SG32-5 & $2800.7 \pm 0.5$ & 25,137 & 33,965 & 5623 & $32,875 \pm 370$ \\
\hline SG32-4 & $2807.0 \pm 0.5$ & 25,242 & 34,070 & 5622 & $32,825 \pm 380$ \\
\hline SG32-2 & $2813.4 \pm 0.5$ & 25,332 & 34,160 & 5620 & $33,475 \pm 345$ \\
\hline SG32-1 & $2855.7 \pm 0.5$ & 25,997 & 34,825 & 5619 & $33,070 \pm 730$ \\
\hline SG33-4 & $2913.8 \pm 0.5$ & 26,882 & 35,710 & 5626 & $33,270 \pm 680$ \\
\hline SG33-3 & $2920.6 \pm 0.5$ & 26,997 & 35,825 & 5627 & $32,640 \pm 330$ \\
\hline SG34-2 & $2976.3 \pm 0.5$ & 27,942 & 36,770 & 5631 & $34,950 \pm 415$ \\
\hline SG34-4 & $2993.8 \pm 0.5$ & 28,232 & 37,060 & 5632 & $35,140 \pm 415$ \\
\hline SG34-3 & $3012.4 \pm 0.5$ & 28,532 & 37,360 & 5633 & $35,070 \pm 460$ \\
\hline SG35-1 & $3067.0 \pm 0.5$ & & 38,285 & 4515,4516 & $35,560 \pm 340$ \\
\hline SG35-2 & $3132.8 \pm 0.5$ & & 39,350 & 4517 & $36,755 \pm 550$ \\
\hline SG36-1 & $3224.9 \pm 0.5$ & & 40,840 & 4518 & $38,205 \pm 650$ \\
\hline SG37-1 & $3311.5 \pm 0.5$ & & 42,241 & 4545 & $40,210 \pm 820$ \\
\hline SG38-1 & $3367.2 \pm 0.5$ & & 43,143 & 4546 & $41,100 \pm 1800$ \\
\hline SG39-1 & $3428.3 \pm 0.5$ & & 44,131 & $\begin{array}{c}4525,4526 \\
4527\end{array}$ & $41,890 \pm 570$ \\
\hline SG39-3 & $3496.8 \pm 0.5$ & & 45,240 & 4528,4529 & $42,640 \pm 780$ \\
\hline
\end{tabular}

